\title{
Pushing the (Visual) Narrative: the Effects of Prior Knowledge Elicitation in Provocative Topics
}

\author{
Jeremy Heyer \\ Indiana University-Purdue \\ University Indianapolis \\ jbheyer@iu.edu
}

\author{
Nirmal Kumar \\ Raveendranath \\ Indiana University-Purdue \\ University Indianapolis \\ niraveen@iu.edu
}

\author{
Khairi Reda \\ Indiana University-Purdue \\ University Indianapolis \\ redak@iu.edu
}

\begin{abstract}
Narrative visualization is a popular style of data-driven storytelling. Authors use this medium to engage viewers with complex and sometimes controversial issues. A challenge for authors is to not only deliver new information, but to also overcome people's biases and misconceptions. We study how people adjust their attitudes toward (or away from) a message experienced through a narrative visualization. In a mixed-methods analysis, we investigate whether eliciting participants' prior beliefs, and visualizing those beliefs alongside actual data, can increase narrative persuasiveness. We find that incorporating priors does not significantly affect attitudinal change. However, participants who externalized their beliefs expressed greater surprise at the data. Their comments also indicated a greater likelihood of acquiring new information, despite the minimal change in attitude. Our results also extend prior findings, showing that visualizations are more persuasive than equivalent textual data representations for exposing contentious issues. We discuss the implications and outline future research directions.
\end{abstract}

\section{Author Keywords}

Narrative visualization; debiasing; persuasion; belief elicitation.

\section{CCS Concepts}

-Human-centered computing $\rightarrow$ Empirical studies in visualization; Information visualization;

\section{INTRODUCTION}

Data-driven storytelling is a popular way of engaging people with complex issues, enabling readers to directly explore the numbers that underlie a story. Authors of these experiences often mix interactive visualizations with textual and rhetorical narratives in an effort to persuade readers. Data journalists have utilized narrative visualizations to address contentious topics, such as racism [3] and climate change [57].

Although such experiences can be visually appealing, empirical studies of narrative visualizations have shown limited Permission to make digital or hard copies of all or part of this work for personal or classroom use is granted without fee provided that copies are not made or distributed for profit or commercial advantage and that copies bear this notice and the full citation on the first page. Copyrights for components of this work owned by others than the author(s) must be honored. Abstracting with credit is permitted. To copy otherwise, or republish, to post on servers or to redistribute to lists, requires prior specific permission and/or a fee. Request permissions from permissions@ acm.org.

CHI'20, April 25-30, 2020, Honolulu, HI, USA

(C) 2020 Copyright held by the owner/author(s). Publication rights licensed to ACM. ISBN 978-1-4503-6708-0/20/04 . .\$15.00

DOI: https : //doi .org/10.1145/3313831. 3376887 success in engaging readers and challenging their assumptions [8,9]. At issue are, perhaps, two concerns: First, most visualizations still present the data in an impersonal manner, without relating to a viewer's prior knowledge or background. Designers typically allow viewers to freely explore visualizations for personal insight. Yet, the reader could still be left unsure how the data relates to them. For instance, it is difficult to draw a link between rising average global temperatures and one's own lived experience of the local weather [66], which can decrease the persuasiveness of a visualization that addresses the global climate crisis. The second issue is that some viewers may harbor implicit biases and misconception that run contrary to the intended narrative. For example, a reader could be influenced by climate change denial [24] or by xenophobic tendencies $[22,30]$. Such implicit biases can block people from effectively processing and internalizing the data-content of a visualization, potentially reducing its impact.

A potential way to addresses these two challenges is to stimulate the reader into reflecting about their own beliefs prior to considering new data. Visualization designers have started experimenting with this idea. For instance, Aisch et al. present viewers with an initially blank line chart, inviting them to predict and sketch the likelihood of a person attending college based on their parents' income [1]. The actual relationship is then visualized alongside the sketch, allowing the reader to contrast their prediction with real data. Researchers began exploring a design space for externalizing viewers' expectations in visualizations [16, 17, 34]. Eliciting prior knowledge while providing feedback on the accuracy of those beliefs seems to improve recall, even among those with little expertise in the subject matter [34]. These results suggest improved cognition when readers are prompted to predict the data prior to seeing it. Could this kind of intervention lead to a debiasing effect that goes beyond memorability? At a deeper level, eliciting priors challenges viewers to concretely articulate an attitude towards an issue. By showing the data-manifestation of people's viewpoints side-by-side with real data, we can surface implicit biases viewers may unknowingly harbor, and potentially persuade them to overcome preconceived notions.

We study the impact of prior knowledge elicitation on attitude change, particularly in contexts that might be riddled with biases and aversions. For our study, we developed a narrative visualization that addresses the ongoing opioid drug epidemic in the US. The visualization provides data and rhetoric to dispel common misconceptions, such as the inefficacy of needle exchange programs $[21,61]$. These misconceptions diminish 
public support for effective harm reduction measures [50, 13], providing an ideal context to study the role of visualization in mitigating prejudice. In a crowdsourced experiment, we assess participants' change in attitude after viewing a custom-made narrative experience. We find that presenting data graphically, as opposed to textually, leads to significantly higher persuasion. However, externalizing participants' prior beliefs in both modalities leads to very small, non-significant attitudinal change over baselines. Qualitative analysis shows that prior specification and feedback is associated with higher incidence of data cognition and learning, but that these did not necessarily increase the persuasiveness of the narrative. We discuss our findings and outline future research directions.

\section{RELATED WORK}

Research into data visualization has historically grappled with how to accurately represent quantitative information [15, 49]. Visualization creators were primarily concerned with facilitating the visual analysis of data [68, 58]. However, the popularization of visualization authoring tools $[7,64,42]$ and their adoption by a broader audience have spurred new applications. Emerging communities of practice, such as data journalism [29], have shifted the focus from analytic to communicative uses [65, 32]. And unlike InfoVis, which was largely conceived in research labs [15], narrative-driven visualizations had developed somewhat organically within various communities. Its roots can perhaps be traced back to the work of Infographics designers [40], who often embellished data charts with elaborate designs as a means to engage and persuade audiences [31]. By the mid-2000s, dynamic visualizations appeared regularly alongside articles on news media websites. Authors began to explore increasingly elaborate designs, combining journalistic elements with data graphics. The emerging stories exhibited a distinct flavor of visualization, complete with its own set of practices and guidelines [27, 70 , 38]. Whereas traditional visualization schools emphasize the objective and abstract presentation of data [73], narrative visualizations subsume the author's own framing, often not shying away from using rhetoric to push a certain narrative [32].

Researchers have analyzed narrative visualizations in the wild to understand this design space. Segel and Heer developed taxonomies of design strategies for storytelling with data [65]. This included techniques (e.g., visual highlighting) and structures (e.g., the 'Martini-Glass' and Interactive Slideshows) designers have used repeatedly. Hullman and Diakopoulos introduced a notion of visualization rhetoric by intersecting the concepts of framing, bias, and user design [32]. Their framework allows authors to prioritize specific interpretations. Figueiras collected common design elements through a focus group, and accordingly provided suggested strategies [25]. We leverage some of these suggested styles in our intervention, adopting 'editorial' elements (e.g., typographic emphases) and procedural rhetoric (e.g., animation and slideshows) to guide viewer attention. Beyond these established design patterns, we are interested in new interactions that might help people relate better to the data, especially in narratives that deal with complex issues.

\section{Empirical Research into Communicative Visualization}

With the proliferation of visualizations and Infographics on the web, researchers have sough to study their communicative impact. Bateman et al. compared visually embellished charts against minimalist versions that displayed the same data without embellishments [4]. Their results suggest that tasteful, non-data elements help people remember the contents and 'messages' of charts, without necessarily sacrificing perceptual accuracy. Wang et al. compared Infographics with data comics [74] - the latter employs comic-inspired panels to expose data sequentially [77]. Their results show data comics to be more enjoyable and comprehensible, possibly due to the linear story structure. Similarly, Zhi et al. show that a 'slideshow' layout is more comprehensible, especially if the textual and data elements can be linked [78]. However, McKenna et al. suggest that the precise manner of control over story presentation (e.g., stepping through discrete slides vs. continuous scrolling) has less impact over engagement than the style of visual feedback (e.g., abrupt vs. animated transitions) [48].

Boy et al. showed that adding narrative elements into exploratory visualizations did not necessarily increase user engagement [8]. In a different study, the inclusion of anthropomorphized characters into data graphics did not affect reader empathy in an otherwise emotionally charged topic about Syrian refugees [9]. These studies suggest that the rhetorical impact of a visualization is not necessarily associated with its interactivity or representational vividness. Kong et al. evaluated the effects of biased visualization titles, and found them to drastically affect the messages people derive from charts [39]. While simply presenting data in visualizations makes them more persuasive [52, 67], people's prior beliefs may play an important role in deciding which message they pay attention to. To achieve communicative impact, authors may need to overcome existing misconceptions among their audience. Our work investigates how to mitigate the effects of such biases.

\section{Prior Beliefs in Visualization}

People rely extensively on their prior knowledge when making sense of new information [37, 35]. Existing frames are often recalled and co-opted to fit new data, more readily than fresh frames can be spontaneously created [36]. It should therefore be easier to communicate data and messages that are congruent with one's beliefs, or that do not conflict with beliefs. This is generally the case in relatively neutral topics, where viewers are not expected to have strong opinions [52]. Yet, there is often a sense of urgency among journalists to address consequential and contentious issues with visualizations (e.g., racism [3] and climate change [57]). These issues can be polarizing, and some viewers may approach them with deeply ingrained misconceptions. It is unclear what persuasive effect if any visualizations can have here.

Although most visualizations present data irrespective of user beliefs, authors have begun to explore alternative designs that engage a viewer's prior knowledge. For instance, in a series of charts exploring how the Obama presidency affected the economy, immigration, and healthcare spending, the reader is invited to predict and sketch how these indicators have changed [53]. The actual trendlines are then revealed to the 


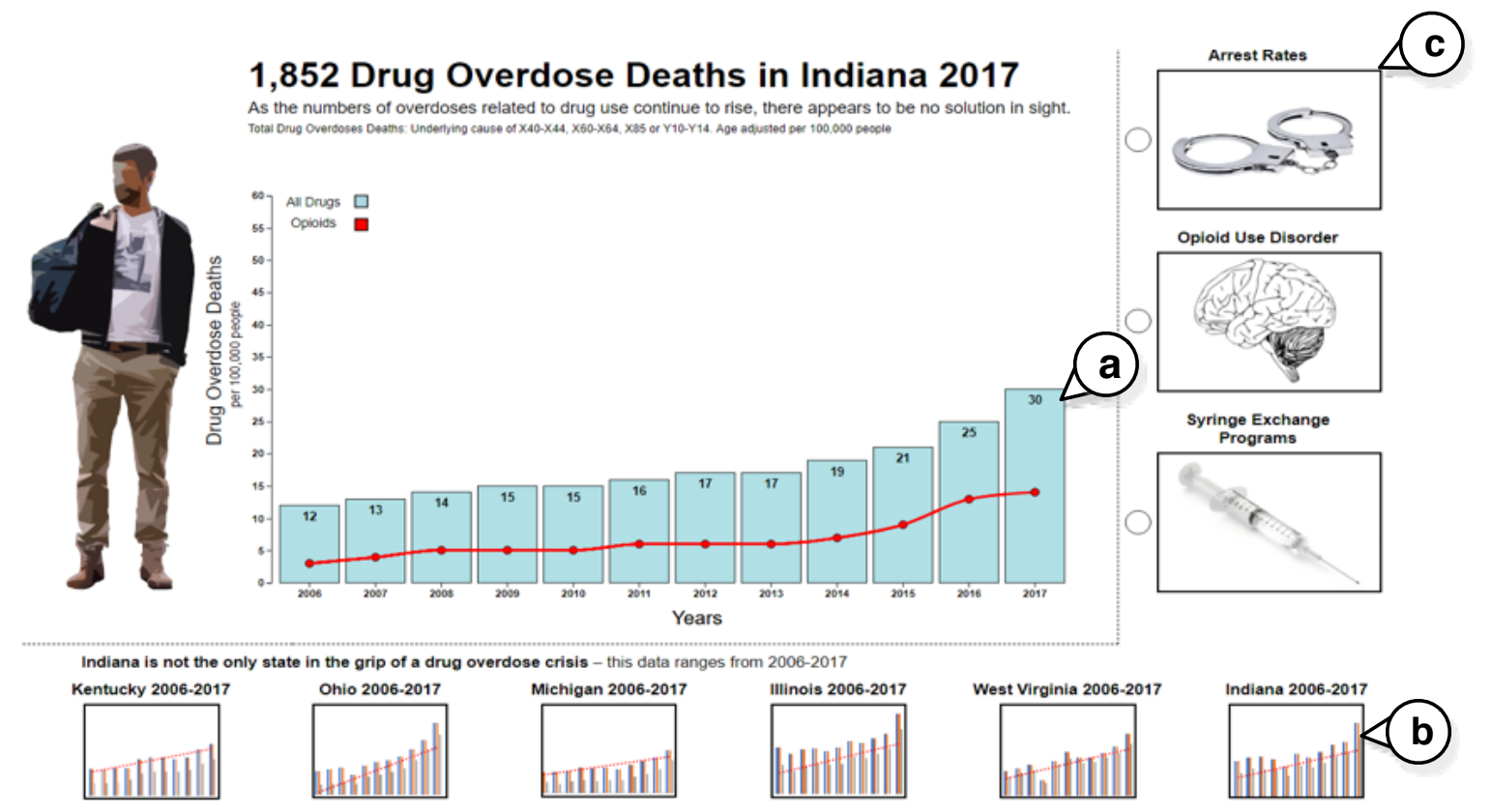

Figure 1. The landing page provided an introduction to the topic by showing opioid-related overdose rates for one state (defaults to Indiana) over time (a). Rates for other states can be seen by hovering over the mini charts (b), which updates the central view. The experience comprises three themes on criminal justice, opioid addiction disorder, and syringe exchange programs, which are viewed one at time by selecting from a right-side panel (c).

reader and visualized alongside their sketch. Kim et al. experimentally tested this kind of graphical belief elicitation, and showed improved data recall amongst participants who were first asked to predict the data [34]. This positive learning effect was attributed to making the gap between one's knowledge and reality more salient, thereby prompting self-correction. Choi et al. studied if this style of interaction can be incorporated into general-purpose visualization tools $[16,17]$. They found that people frequently opt to test their beliefs, by going through the extra step of first predicting the data. We build on these findings, and study the persuasive effect of engaging the prior knowledge of viewers in narrative visualizations.

\section{RESEARCH QUESTIONS}

Our primary goal is to improve engagement with narrative visualizations. We are particularly interested in methods for delivering the supporting data so as to increase persuasiveness of the experience, especially when the author is exposing provocative topics. Engaging the prior knowledge of the audience is one way of increasing engagement. It also provides the author with an opportunity to highlight places where one's mental model disagrees with the data [16]. Prior work has demonstrated benefits to this kind of engagement, such as increased recall [34]. However, incorporating people's beliefs into visualizations may also have a further-reaching debiasing effect, which can be highly useful when authors discuss contentious and potentially polarizing issues. Here, viewers may come to the experience with deeply ingrained notions that make it difficult to depart from existing beliefs, even when presented with data to the contrary. However, if we are careful to present such data in a way that contextualizes existing beliefs, we may have a higher chance of persuading minds.
Because we are interested in evaluating the persuasive potential of data narratives, we move beyond metrics of recall [39, $34,4]$. Instead, we adopt Pandey et al's notion of attitudinal change, and measure how people use information and facts to update their beliefs towards an issue [52]. We expand on that work by using a pre-validated multi-item survey, as opposed to a metric derived from a single question. This allows us to track a wider encompassing notion of attitudinal change, wherein data facts and measures are not necessarily in one-to-one correspondence. We pose the following research questions:

RQ1 - Does eliciting beliefs from people and providing feedback on the accuracy of those beliefs increase the persuasiveness of data-driven narratives? In other words, are people persuaded more easily by the author's message when the supporting data is contextualized to their beliefs and explicitly compared against their prior opinions?

RQ2 - Does the effectiveness of prior specification depend on the representation medium? We specifically compare graphical representations, where data is shown in charts, against textual representations that integrate data directly into the narrative. Visualizations are generally thought to be more persuasive than text $[23,52]$. However, by weighing the potential payoff of these two design factors, we we can provide more practical guidelines for authors.

\section{CONTEXT AND INTERVENTION}

To answer the above research questions, we designed a narrative visualization that centers around the opioid epidemic in the United States. We specifically featured Indiana and the surrounding states (Kentucky, Ohio, Michigan, Illinois, and Western Virginia), which have recently experienced exceedingly high overdose rates stemming from opioid addiction [63]. 


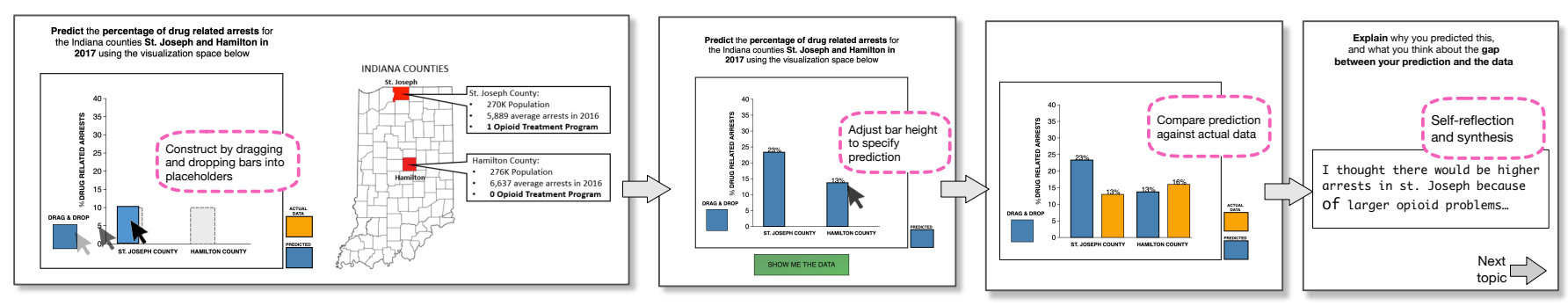

Figure 2. Illustration of the steps participants followed to specify their predictions graphically by 1) 'constructing' bars in data placeholders, 2) adjusting bar heights to specify prediction, 3) seeing their prediction contrasted with actual data, and 4) providing an open-ended reflection.

For instance, Indiana ranked 13th in the number of opioid overdose deaths per capita [11]. Current data identifies over 3.4 million distinct opioid prescriptions in Indiana, which amounts to an astonishing rate of approximately one prescription for every two residents [28].

Reports on the volume of opioid use and addiction are prevalent topics in news media. These reports can create negative perceptions [18] and potentially worsen the problem [20]. Treatment options often involve the creation of new infrastructure (e.g., treatment centers or sober living facilities), which sometimes require new legislation along with support from local communities. However, given the highly stigmatized and sensitive nature of addiction [47], people sometimes harbor counter-productive beliefs or misconceptions about impacted populations [50, 12]. For instance, many believe that a sober living facility in a community will results in increased crime and drug use [56] and that implementing a syringe exchange program (i.e., a harm reduction approach) will increase drug use and result in an influx of discarded syringes [21]. Misconceptions like these can translate into policy resistance at the local level, which makes the adoption of otherwise effective treatment or harm reduction initiatives less likely $[60,69]$. Yet, these issues provide an ideal context for our research.

Our narrative visualization aims to clarify some of the misconceptions surrounding opioid addiction and treatment. We sought to challenge implicit biases by providing curated but real data, and subsequently test if we can affect a shift in people's attitude towards substance use disorder. In particular, our narrative visualization addresses two issues: the disassociation between criminal justice and opioid use [26], and the generally negative attitude towards harm reduction measures, such as syringe exchange programs, which have historically had low levels of support in the US [47]. For visualizations, we opted for familiar plots (e.g., bar and line charts).

\section{Narrative Visualization}

This section describes the narrative visualization we designed and subsequently used in an experiment. The visualization consisted of a landing slide (see Figure 1), which introduces the viewer to the topic by painting a broad-brush picture about the scale of the opioid epidemic in the US. The landing slide consisted of a central bar chart illustrating the rising trend of deaths attributed to opioid overdoses. Six smaller charts were displayed below, each depicting numbers for a different state. Upon hovering over the bottom charts, the central chart is updated to reflect the selected state. The experience comprised three subtopics addressing the following themes: 1) the disassociation between crime and treatment centers, 2) the classification of opioid addiction as a medical disorder, as opposed to a criminal justice issue, and 3) the effect of harm reduction programs, specifically, syringe exchange programs. Syringe (or needle) exchange programs typically allow people to obtain new sterile syringes in exchange for used ones in an effort to reduce transmission of blood-borne diseases. The three themes, which are accessed from the landing slides, were chosen given the generally negative portrayal in the media, misconceptions, and overall lack of public enthusiasm for treatment infrastructure $[6,26]$

The design in each theme followed an Interactive Slideshow structure [65]: each theme consisted of a number of slides (between 3-5) shown in a sequence. Viewers stepped through the slides at their own pace using next and back buttons. The slides provided a combination of contextual and rhetorical arguments (e.g., that there is little to no association between arrest rates in a community and the presence of drug treatment centers), while also furnishing quantitative data in support of those arguments. For instance, in the crime and safety theme, the viewer is shown data contrasting drug-related arrests between two Indiana counties of similar populations but a different number of addiction treatment centers. The data demonstrates no correlation between the number of arrests and the presence of a treatment program in that community. Themes two and three provide data showing that opioid use disorder is a diagnosable medical condition and that syringe exchange programs do not promote drug use, respectively. The conclusion of each theme consisted of a 'synthesis' slide, summarizing the author's main points and reiterating key facts from the theme, after which the viewer is returned to the landing slide. The narrative experience is completed after the viewer steps through and completes all three themes. The order of theme presentation is decided by the viewer.

\section{EXPERIMENT}

To answer our research questions, we conducted a crowdsourced study using the narrative visualization described above as stimulus. Using a survey, we collected participant attitudes prior to and after the narrative experience. We then measured the extent to which participants update their attitudes and align their beliefs with the narrative.

\section{Study Design}

We studied two factors: Interaction technique and data representation Medium. We tested two kinds of interactions: 


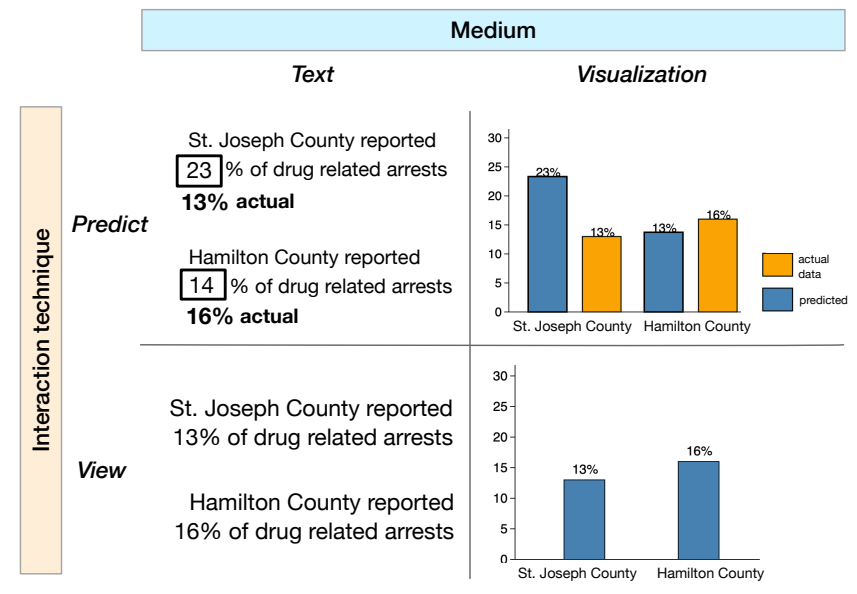

Figure 3. Illustration of how participants viewed or interacted with the narrative's data in the four experimental conditions. The conditions reflect a factorial design of two Mediums (Visualization or Text) and two Interaction techniques (Predict or View).

Predict and View. In Predict, we asked participants first about their beliefs by prompting them to predict portions of the data (e.g., on arrest rates around treatment center) via specially designed interactions. After 'drawing' or typing their predictions, participants viewed the actual data side-by-side with their predictions. In View, participants immediately saw the relevant data with no opportunity to externalize their beliefs, as in most visualizations. The second factor, Medium, also consisted of two levels: Visualization or Text. In the Visualization condition, data was shown in the form of bar charts, whereas the Text condition presented the data numerically and embedded within the narrative prose. Both Visualization and Text included identical textual arguments that were displayed on the same slide as the data, but the two conditions differed in the manner in which the quantitative data was delivered.

We employ a $2 \times 2$ factorial design and investigate all combinations of Interaction techniques and data representation Mediums. This design yields four unique experimental conditions (illustrated in Figure 3):

* View-Visualization: Quantitative data was shown graphically in bar charts, with contextual and supportive arguments information on the left side of each slide. For example, in the theme on syringe exchange programs, participants viewed data reflecting the percentage of diagnosed HIV cases in Indiana that were linked to intravenous drug use. The supportive arguments provided contextual information about syringe exchanges and their efficacy.

* View-Text: Data was presented numerically (e.g., as percentage points) alongside supportive arguments. For example, in theme on syringe exchange programs, participants viewed the same data shown in the visualization conditions, but that data was provided textually.

* Predict-Visualization: Data was shown graphically in bar charts. However, the charts were initially blank; participants were first prompted to predict the content of the charts before the data is revealed. For instance, in the crime and safety theme, the chart consisted of a Y axis labeled 'Drug related arrests' and an X axis consisting of two groups: 'St. Joseph County' vs. 'Hamilton County'. The two bars for these two counties appeared as greyed-out placeholders, initially. Participants dragged a blue square onto either placeholder to 'construct' a bar, and then adjusted the height of that bar to specify their prediction of arrest rates in each county. Participants then clicked a 'Show me the data' button, causing the actual data to appear (via animated transition) in adjacent orange bars. This sequence is illustrated in Figure 2.

* Predict-Text: Data was also presented textually in this condition. However, before the data is revealed, participants were asked to provide a prediction by filling in blank textboxes. For instance, in the arrest rates theme, participants were instructed to "Predict the percentage of drug related arrests" in two counties. The slide displayed two empty place holder text-boxes (e.g., "St. Joseph county reported [blank]\% of drug related arrests"). Upon entering predictions and clicking a "Show me the data" button, the real quantities were revealed beneath the predicted values (see Figure 3 ).

The knowledge content in the four conditions was identical, with the difference being the data representation Medium ( $\mathrm{Vi}$ sualization vs. Text) and the Interaction technique (Predict vs. View only). Moreover, all conditions required participants to explain the data and/or justify their predictions, by typing into a text-box. These prompts instructed participants to "Explain what you think the data shows", and whether the data "was different than what [they] had thought." Participants were also prompted to reflect on the entirety of a theme at the concluding synthesis slide, and describe what they had learned. The prompts served two purposes: 1) self-explanation can be an effective meta-cognitive strategy to increase learning [2, $5,59]$, and 2) the explanations enabled us to capture nonstructured responses from participants, which we later analyze to understand their reasoning processes.

\section{Survey Development}

We developed a survey to capture prior attitudes and measure belief change among participants. Our survey was largely adapted from items in the Drug and Drug Problems Perceptions Questionnaire (DDPPQ), which were modified slightly to fit the themes of the narrative [76]. The DDPPQ survey was primarily designed to measure the attitude of mental health workers towards drug users in order to assess training needs. The instrument has been validated in multiple studies, showing good internal consistency and relevance [75]. Although originally intended for healthcare professionals, the survey has been tested on non-professionals (e.g., students [44] and teachers [55]). In addition to the original DDPPQ items, we adapted two questions from Lowder et al's study on the attitude of criminal justice professionals about substance use and mental illness [45].

The survey comprised of 16 items in total. For each item, participants were asked to indicate their agreement with a certain statement using a 5-point Likert scale. The choices available comprised a divergent 'Strongly Disagree' to 'Strongly Agree' scale, with a 'Neutral' point at the middle. Survey items covered five topics corresponding to stigmatizing issues on 
addiction and treatment [62]. Questions 1-3 dealt with perceptions of crime and safety around treatment centers for drug addiction (e.g., Areas surrounding addiction treatment centers are safe areas). Questions 4-6 dealt with the perception of opioid use as a diagnosable disorder, as opposed to a criminal justice problem (e.g., Opioid use disorder is a diagnosable disease). Questions 7-9 were about syringe exchange programs, their effectiveness, and acceptance (e.g., I can accept a syringe exchange program in my community). Questions 10-12 asked participants about their willingness to support legislation on drug addiction (e.g., I would support laws and legislation written to support those with drug addictions). Lastly, questions 13-16 asked about public funding for treatment infrastructure and harm reduction measures (e.g., Public funding should be increased to reduce drug addiction). Two attention questions comprising simple arithmetic were randomly inserted into the survey to ensure participant engagement.

\section{Attitude Change}

Participants completed the survey twice: once before experiencing the narrative and once after. Likert responses were transformed into ordinal values $(-2$ to +2$)$, in order to quantify the change in attitude from the pre- to the post-experience survey. This was done by computing an attitude change score for each question (i.e., difference in answer between the two surveys). The sign for three questions was reversed so that positive is always aligned with the narrative's message. Change scores from all 16 survey questions were summed up yielding a net attitude change score for every participant. Positive attitude change is associated with aligning one's view with the message of the narrative.

\section{Procedure}

We employ a between-subject design: participants were randomly assigned to one of the four conditions. After viewing a study information sheet, they first completed the preexperience survey. Participants in conditions that included prior elicitation (i.e., Predict-Text and Predict-Visualization) were provided with a short tutorial allowing them to practice the belief elicitation technique until they felt comfortable. Participants then saw the landing page (Figure 1) and completed the experience as described above (see 'Narrative Visualization'). After completing the third, final theme, participants were directed to the post-experience survey. Lastly, participants completed an exit demographic survey.

\section{Participants}

We conducted power analysis for a two-way ANOVA. Using estimate effect sizes from a pilot, we calculated that 40 participants per condition would give us a power of 0.8 at an alpha of 0.05 . We recruited 160 participants (103 males, 56 females, 1 other) from Amazon Mechanical Turk, with 40 participant per condition. We only recruited subjects who are residents of the US for topic relevance. We excluded those who failed the majority of the engagement questions. New participants were recruited in place of those excluded until we reached our target sample size. Participants had a mean age of 32.4 years. They represented a wide range of educational backgrounds: $28 \%$ completed high school, $14 \%$ have associate degrees, $41 \%$ bachelors, $13 \%$ masters, and 3\% doctorate. Participants completed the experiment in 22.2 minutes on average $(S D=11.8)$. They were compensated with $\$ 3.00$.

\section{Hypothesis \\ We developed the following hypothesis:}

H1 - Eliciting priors from participants and providing feedback on their accuracy increases persuasion. We expect participants in the Predict conditions (i.e., Predict-Text and Predict-Visualization) to exhibit larger attitudinal change, compared to those in the View conditions (View-Text and ViewVisualization). This is because seeing the accuracy of one's beliefs can induce a debiasing effect, by highlighting potentially skewed predictions. Comparing beliefs and data may serve as a form of rhetoric, causing participants (especially those with outlying beliefs) to feel 'pressured' to align their views with the narrative.

H2 - We expect prior specification to induce larger attitude change among participants who view a visualization (PredictVisualization), compared to those who view a textual representation (Predict-Text). This is because feedback on the accuracy of beliefs are probably more salient when presented graphically as opposed to textually [34]. This in turn could cause more participants to reflect on discrepancies between beliefs and data, possibly persuading them to align with the author's narrative.

H3 - We expect visualizations to be more persuasive than text. We hypothesize that participants in the visual medium will exhibit larger attitude change relative to those who view the data as text. This hypothesis is based off earlier studies comparing textual vs. visualized data, which generally found the latter to be more 'informative' [23] or persuasive [52].

The above predictions represent extrapolation of earlier results $[52,34]$. However, we seek to test these predictions in a new communicative context: data-driven narratives that deal with provocative and potentially polarizing topics. Therefore, while we initially expect the above hypotheses to be borne out, it is possible for the context to significantly change (e.g., diminish) the effects sought. Therefore, in addition to testing our predictions, we also aim to quantify the relative impact of the main two factors (prior specification and representation medium). As a secondary objective, we aim to provide qualitative insights on how participants engage, whether cognitively or affectively, with the data-content and argumentative elements of the narrative.

\section{Quantitative Results}

We first analyze attitude change from the pre- to the postnarrative survey. We then explore participants' open-ended responses. ${ }^{1}$

\section{Attitudinal Change}

A Shapiro-Wilk test indicates the resulting change scores are normally distributed $(p=0.1)$. We first confirm whether participants internalized the contents of the experience. T-tests

\footnotetext{
${ }^{1}$ Data from this study is available at: https://osf.io/2u95t/
} 


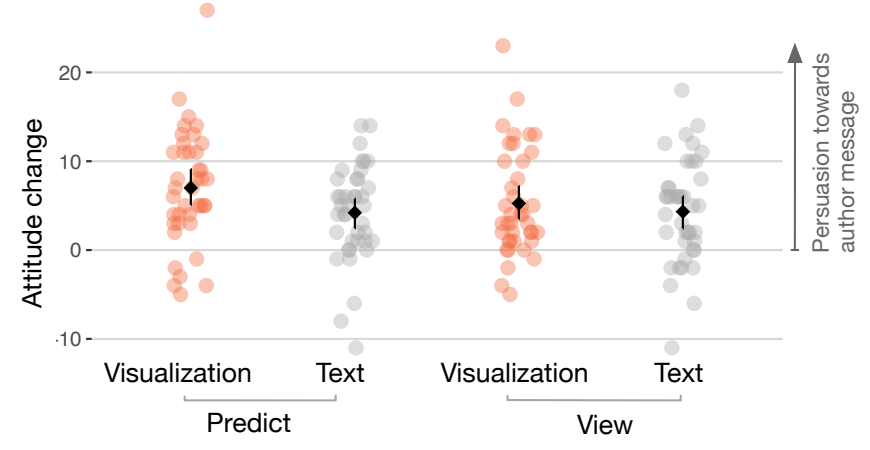

Figure 4. Attitudinal change by condition. Each dot represents one participant. Diamonds depict group average ( \pm bootstrapped 95\% CIs)

confirm that all four conditions (independently) produce positive, non-zero attitude change ( $p<0.001$, with Bonferroni correction).

We employ a $2 \times 2$ ANOVA model to test the impact of the two factors on attitude change. The first factor denotes the Interaction technique (with two levels, Predict or View) whereas the second denotes the data representation Medium (Visualization or Text). Figure 4 shows the resulting attitude change in the four experimental conditions. We did not find a significant main effect of Interaction technique $(F(1,156)=0.751, p=0.3876)$. Participants who were asked to first predict the data exhibited similar net attitude change (mean change: 5.61 Likert points, $C I$ : [4.23, 6.92]) relative to those who immediately saw the data with no opportunity to specify priors (mean: 4.79, CI: [3.55, 6.07]).

We found a significant main effect of Medium $(F(1,156)=$ $3.944, p<0.05)$. Participants who saw the supporting data as visualizations demonstrated higher alignment with the author's message (mean: 6.12, CI: [4.84, 7.49]) than those who viewed the same data as text (mean: $3.05, C I$ : [3.05, 5.48]). We did not detect interaction between the two factors $(F(1,156)=0.999, p=0.319)$. The results can be explained by independently considering the effects of Interaction technique (non-significant) and data representation Medium (significant). Figure 5 shows averages for the two main factors.

\section{Effect Sizes}

We complement the above analysis with estimations of effect sizes. We compute Cohen's d to understand the potential extent of attitude change for the two factors, as well as within the Medium factor. Figure 6 illustrates the resulting effect sizes. The top two estimates represent the main effects of representation Medium (Visualization vs. Text) and Interaction technique (Predict vs. View), respectively. Visualizing the data component of the narrative is likely to have a moderate effect on persuasion (estimated effect size: 0.314, CI: [0, 0.628]), but the effect can be as high as medium or as low as to be almost negligible. On the other hand, eliciting priors and providing feedback on belief accuracy appears to have a very small effect on attitude change (est: 0.136, CI: $[-0.177,0.448]$ ), but with a potentially moderate-to-medium effect on the upper end.

Lastly, we analyzed the impact of prediction in Visualization and in Text separately. The bottom two estimates in Figure 6
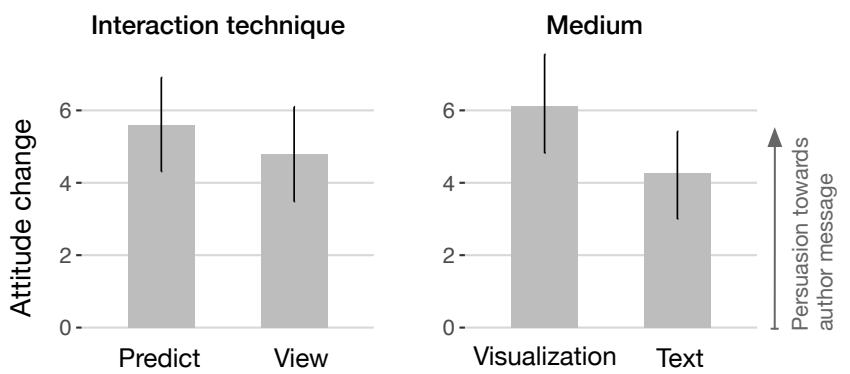

Figure 5. Average attitudinal change by Interaction Technique (left) and Medium ( \pm 95\% CIs)

show the effect of specifying priors vs. immediate viewing of data in visualizations (second to bottom estimate), and in text (bottom). Confidence intervals for these latter two estimates were adjusted for multiple comparisons. Prediction in visualization has a potentially small effect (est: $0.281, C I$ : $[-0.232$, $0.795]$ ). However, there appears to be a large variation in how individuals are affected by visualizing priors. Therefore, the effect is likely to be small and potentially unreliable at the population level. In Text, however, prediction appears to have a negligible impact on attitude (est: $-0.022, C I:[-0.533$, $0.489]$ ), while allowing for a wide margin of outcomes.

Overall, results show that visualization is a moderately more persuasive medium. Encoding data graphically, as opposed to textually, should result in moderately higher attitude change. By comparison, the impact of prior specification, wherein people are asked to predict what the data might show before seeing it, should yield only a very small communicative impact.

\section{Qualitative Results}

We examined the thought processes of participants by analyzing their qualitative reflections. Throughout the narrative, participants were prompted to respond to each data displays (8 in total) with open-ended textual entries. These responses contained explanations of participants' understanding of the data, as well as reflections on gaps between the data and their expectations. In analyzing these responses, our goal was to shed light on how participants interacted with the narrative, and on the cognitive or affective processes they drew upon to update their attitude. Altogether, we obtained 1,280 openended responses, which we segmented [71] and coded using inductive thematic analysis [10]. We grouped like responses

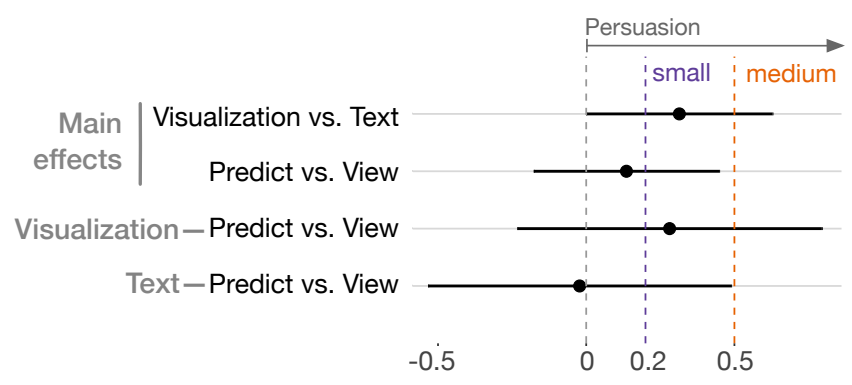

Figure 6. Effect sizes (Cohen's d) for the main effects (top two estimates), and for the effect of prediction separately under visualization and text ( $\pm \mathbf{9 5 \%}$ CIs, corrected for multiple comparisons). 


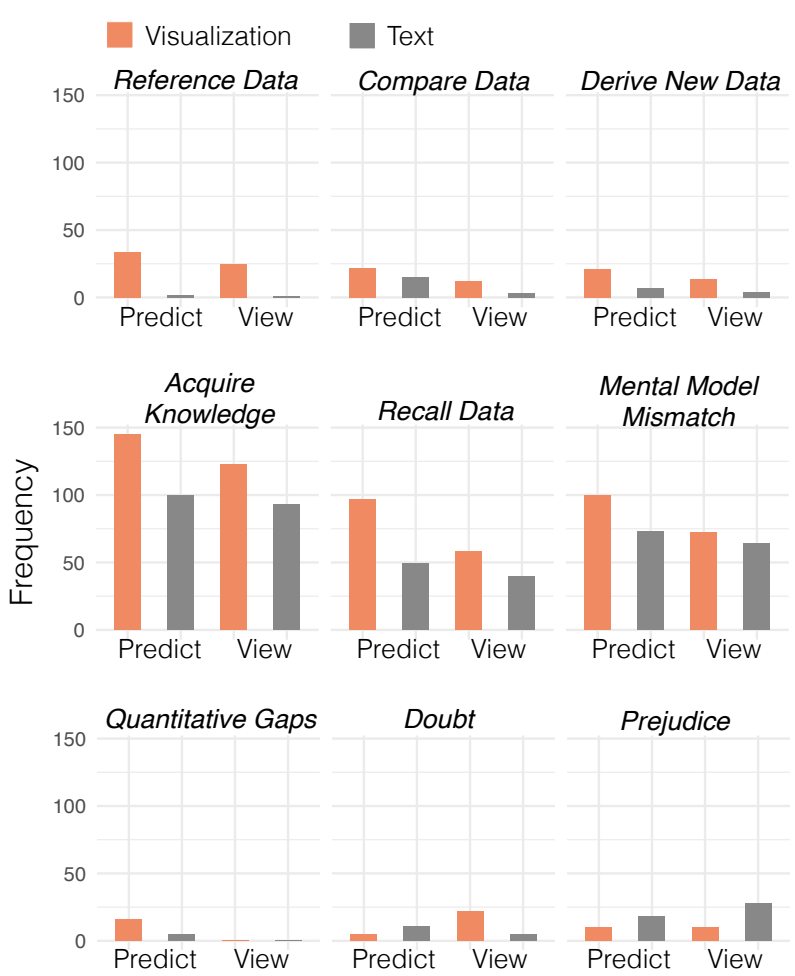

Figure 7. Frequency of the nine qualitative themes broken down by condition.

until themes began to emerge, and iteratively revised our coding scheme. We ultimately identified nine recurring codes which we further organized under four distinct themes: Data Cognition, Learning and Recall, Mental Model Discrepancy, and lastly Doubt and Prejudice. We discuss these four emerging themes and provide examples from the study. Figure 7 shows code frequency across the four experimental conditions.

\section{Data Cognition}

The responses frequently contained evidence of data cognition mechanisms at work. This includes instances where the participant would explicitly Reference Visual or Textual Data (62) or, less frequently, Compare Data they had encountered in the narrative (52). For example, when textually predicting the number of opioid prescriptions in Indiana, Participant \#374 compared their prediction to the real data: "based on the previous data that was provided, I thought the answer was 109 active painkiller prescriptions for 100 people. However, the number for 2017 is 74 and is less than the current 109."

Additionally, we came across instances of Deriving New Data (46). Here, a participant would start from the data provided in the narrative, and use that data to compute new quantitative information. For example, when asked about newly diagnosed cases of HIV, P243 explained their knowledge of HIV transmission and then used quantitative data from the narrative to calculate the difference between different transmissions methods. "The data shows that $32 \%$ of the newly diagnosed HIV patients probably shared a needle... I thought sexual transmission would not have been $68 \%$ of the cases." It seems that data-cognition processes were frequently invoked by participants to either highlight or generate new quantitative 'evidence'. This evidence was then used to support their explanation or to provide rationale for their prediction.

\section{Learning and Recall}

We frequently found comments where participants would explicitly indicate they had learned something new from the narrative, or had their existing knowledge corrected. This code identified as Acquiring Knowledge had the highest frequency (461) and revolved around specific cases of self-identified learning. In one case, P270 was asked to reflect on the criminal justice topic, specifically how an opioid treatment program impacts drug related crimes in a community. "I learned that having an opioid treatment facility in your community does not increase drug related crime in the area." Similarity, P364 used new knowledge to correct an existing notion they had about HIV transmission: "the percentage of HIV was lower than I thought... I thought needles [compared to sexual transmission] would have been a higher percentage." Learning occurred consistently across the four experimental conditions, but appeared to happen more frequently in the two Visualization conditions.

Responses also seemed to contain evidence of Recalling Data, where a participant would recite quantitative information they had encountered earlier in the narrative (244). Recalled data was often used to justify predictions or to generate new inferences. For example, P247 explained their prediction by recalling a previously presented statistic on the number of HIV infections attributed to drug use: "I thought sharing needles was the number one reason HIV was being spread [but] $32 \%$ suggests that while sharing needles is a problem, HIV is still likely to be spread by other means." Speculatively, recalled data may provide a kind of rationale for participants to change their beliefs, or, at minimum, appears to indicate acceptance of the narrative information as a valid source of data to base one's prediction off of.

\section{Surprise and Mental Model Mismatch}

Participants frequently exhibited surprise at the data presented, suggesting that the data did not fit their existing beliefs. A Mental Model Mismatch was noted consistently throughout the conditions, but with a notably higher rate in the Predict-Visualization condition (see Figure 7). This response also represented the second most frequently recurring code (309) after Acquired Knowledge. In one example, a participant expressed surprised at the lack of correlation between Indiana's rate of prescription painkillers with its presumed lower economic status: "[Prescription rates] is different than what I thought, because I would have thought it would be higher due to the poor economy in Indiana" (P322). Another participant stated their surprise at a statistic indicating that all patients undergoing formal substance use treatment would had been medically diagnosed with substance use disorder: "I did not realize that [opioid use disorder] would be 100\% of people receiving treatment. I would not have thought this definition applied to everyone in treatment" (P364).

In addition to expressing general surprise, participants attended to specific Quantitative Gaps between their expectation and the data (21). For example, P300 discussed the gap between their prediction of opioid use disorder diagnoses 
and those diagnosed in treatment. "I knew it would be a high percentage [of persons receiving treatment and who are medically diagnosed], but I did not realize it would be $100 \%$. I think the gap is just my lack of knowledge." In this example we see the recognition of a knowledge gap and a willingness on the part of the participant to reconcile their knowledge with the narrative information presented.

\section{Doubt and Prejudice}

There were relatively few instances of participants expressing Doubt (43) and questioning the veracity of the data. For instance, P428 disputed our data on the relationship between crimes and drug treatment centers: "I don't agree with it. It says there is no relationship between opioid programs and crime, however many years of firsthand experience tell me otherwise." We also came across a significant number of comments that might indicate Prejudice against a certain group of people (66), such as HIV patients: "[HIV rates] not too surprising, HIV is a disease for self-destructive people” (P386).

\section{DISCUSSION}

It is often argued that the value of visualizations lies in their ability to compel viewers to see the unexpected $[14,15]$. This is not only advantageous in exploratory settings, but can also be highly useful in communicative visualizations, especially when authors address contentious issues. Viewers are likely to bring pre-existing notions when viewing such visualizations, and some of these beliefs may be unfounded. Eliciting prior beliefs from viewers and visualizing those beliefs alongside data can, in theory, prompt deeper reflection onto potential biases and misconceptions. To test this hypothesis, we measured how much viewers update their attitude after viewing a datadriven narrative on the US opioid epidemic. We tested four different ways of presenting the supportive data, including the use of textual or graphical representations, and interactions that prompt participants to externalize their beliefs prior to revealing data, or that simply show the data immediately. We measured attitude change using a pre-validated survey instrument [76], and analyzed qualitative responses provided by participants throughout the experience.

\section{Attitude Change in Provocative Topics}

We first hypothesized that eliciting prior expectations (e.g., about the incidence of drug-related crimes around treatment centers), and giving participants feedback on the accuracy of those expectations, would result in higher persuasion. The rationale is that articulating predictions and then seeing those predictions alongside actual data would serve to highlight flaws in one's beliefs, prompting self-correction. Results show that prior elicitation does not have a significant effect on attitude change. Specifically, participants who first predicted the data (whether graphically of textually) where no more likely to register a positive change in our survey than those who simply viewed the data. The effect of prediction was estimated to be very small (Cohen's $d=0.136$ ). Thus, we do not find sufficient evidence to support $\mathrm{H} 1$.

We then investigated whether the impact of prediction is more pronounced in visualizations, compared to textual representations (H2). We found no significant evidence of such interac- tion. However, prediction in the visual modality may have a small positive effect over prediction in text $(d=0.281)$. Yet, variations in attitude change among participants was high, leading to wide confidence intervals (CI for $d=[-0.533,0.489]$ ). We thus find no sufficient evidence to support $\mathrm{H} 2$.

These results are somewhat surprising; earlier work suggests that the kind of interactions we tested (e.g., graphical prediction) should improve people's memories of data [34]. In particular, our experimental setup and elicitation method was similar to a study by Kim et al. [34]. This includes asking participants to specify predictions, providing feedback on the accuracy of those predictions, highlighting gaps between beliefs and the real numbers, and finally prompting reflection with self-explanation. Furthermore, all of our conditions where informationally equivalent, providing the same narrative while only varying the manner in which participants saw and/or interacted with the supporting data. Yet, our study does not demonstrate a significant persuasion advantage to incorporating one's predictions in visualizations (or in textual representations, for that matter). It is worthy to note that all tested conditions proved effective at swaying participants towards the message of the narrative. On average, participants responded with positive change in their perceptions of the relationship between public safety and drug treatment centers, and the effectiveness of harm reduction programs. However, the extent of attitude change was largely unimpacted when participants shared their prior knowledge, and saw their beliefs contrasted with empirical data.

One potential factor to these non-significant results is the relatively large variation among individuals, which may reflect differences in willingness to changing belief. In particular, there appears to be a wide distribution of attitude change scores (see Figure 4). This is expected, considering that individual differences play an important (yet not fully understood) role in how people reason with visualizations $[19,79,51]$. Differences in background, for instance, may shape the degree to which people trust depictions of data $[54,46]$ and, perhaps, the extent to which they draw upon such artifacts to update personal beliefs. It is possible that individual differences played a larger role in dictating persuasion, more so than the specific interaction mechanism. Another possible reason is that collecting open-ended reflections from participants may, in and of itself, prompt self-correction and debiasing. All four conditions we tested included prompts for participants to rephrase data in their own words. This may have provided sufficient grounds for attitude change, possibly overwhelming any otherwise smaller persuasion effects.

\section{Visualization vs. Text}

We predicted visualization to produce greater attitude change than text (H3). Results indeed support this hypothesis, replicating earlier findings [52]. However, whereas Pandey et al specifically avoided "polarizing" topics, our study extends that work by showing a similar "persuasive power" for visualizations over text in provocative narratives. There are several reasons why a visualization can be more persuasive than text. For one, graphics can be more salient than sentential representations [41]. The patterns contained in visualizations are 
more readily available to viewers' cognitive processes, which leaves more time for mental integration and reflection. Additionally, and perhaps controversially, visualizations may in and of themselves lend more credibility to data because of their association with science [67]. It may be that surface qualities of data graphics, as opposed to their comprehensibility, make them more (or less) convincing [43]. Overall, the results suggest that visualization are more persuasive than equivalent textual representations, even when the data is steeped in controversial or possibly polarizing issues.

Another noteworthy observation is a reduction in the number of comments that could be deemed prejudicial (coded as Prejudice), when viewing visualizations as opposed to text. We specifically used this code when we came across derogatory expression toward an issue or a population. Although we encountered such sentiments in each experimental condition and within every one of our three themes, prejudice was more frequently associated with the Text conditions. It is unclear why textual data is more conducive to prejudicial comments. However, it is possible for visualizations to invite a more rational engagement with data [23] or summon scientific credibility $[33,67]$. This in turn may inhibit reactionary impulses that might otherwise surface when one's beliefs are challenged. We note that this prejudice pattern was found through an exploratory analysis, so further confirmatory evidence would be needed to corroborate.

\section{Learning vs. Believing}

Participants' reflections suggest that prior elicitation (especially visually) can change the quality of the experience. Participants who graphically predicted the data had the highest frequency of codes indicating knowledge acquisition and data cognition (see Figure 7). Of particular interest are codes associated with learning and recall (Acquiring Knowledge, Recalling Data) and surprise (Mental Model Mismatch, Quantitative Gaps). Comments of participants in Predict-Visualization suggested increased awareness of misconceptions or knowledge gaps held. Their reflections also indicated a greater number of new facts learned. For example, we observed 145 instances of acquired knowledge and 100 reflections on mismatches between one's mental model and the information presented. The frequency of these codes in Predict-Visualization generally surpassed the other three conditions. View-Visualization came in second overall in the frequency of learning-relevant codes.

Overall, the increased incidence of learning and data cognition in Predict-Visualization did not translate to greater attitude change, as measured quantitatively. It is therefore perhaps necessary -especially in provocative narratives especially- to distinguish between learning and persuasion. Although acquiring new facts or even correcting existing information can be necessary first steps, those may not be sufficient to changing one's beliefs about an issue, particularly a contentious one. This tautology is sometimes overlooked, but figures centrally in discourse about 'wicked problems' [66]. For example, a study on climate change perception showed that one's subjective attitude towards meat was associated with willingness to curtail meat consumption, whereas knowledge about the impact of meat production was not [72]. Similarly, our study shows that it may be possible to increase learning and reflection by contextualizing people's prior beliefs in visualizations. However, the extra gain in knowledge may not necessarily persuade people to change their attitudes. Visualization authors should therefore attempt to address two distinct facets to persuasion: informing and changing beliefs. While the visualization literature appears to conflate these two facets, addressing them may require distinct design strategies, along with different evaluation metrics. Future work should attempt to tease out these differences.

\section{LIMITATIONS AND FUTURE WORK}

There are a number of limitations to our study that should be contextualized when interpreting the findings. First, we limited recruitment to subjects who are residents of the US, so as to maximize topic relevance. While subjects had a variety of educational backgrounds, it is uncertain if this crowd-sourced sample is representative of the broader population. Moreover, individuals may also exhibit varying levels of willingness to changing their views and attitudes. Future work could try to address this aspect, for instance, by measuring personality traits that are correlated with adaptability to new ideas.

The specific topic may also play a role in shaping the results. Our goal in spotlighting the opioid epidemic is to expose a contemporary public concern that is riddled with misconceptions and stigma. It would not be surprising for a different topic to yield different results, such as larger (or smaller) attitudinal change. Future studies could focus on the contentiousness of the topic as a factor.

\section{CONCLUSION}

Narrative visualization is a popular style of data-driven storytelling. We investigated ways for increasing reader engagement with this medium, especially when exposing provocative topics. Specifically, we evaluated whether asking people to specify their prior beliefs, and giving them feedback on the accuracy of those beliefs, can increase persuasion. Results indicate that prior elicitation has a likely minimal (non-significant) effect on persuasion. Participants were no more likely to change their attitude when asked to predict the data beforehand, than if they had simply viewed that data. However, consistent with earlier studies, we see qualitative evidence of increased learning and data cognition among the former group. The results, on the other hand, confirm that visualizations are generally more persuasive than equivalent textual representations, and extend those findings to include visualizations about potentially polarizing issues. Our findings suggest that designers should regard informing and belief changing as potentially separate outcomes of interacting with visualizations. The results also call for additional research on how to better persuade audience with narrative visualizations.

\section{Acknowledgments}

We would like to thank Brad Ray for his help in designing the survey, and Taylor Childers for assistance in developing the visualization. This paper is based upon work supported by the National Science Foundation under award 1755611. 


\section{REFERENCES}

[1] Gregor Aisch, Amanda Cox, and Kevin Quealy. 2015. You Draw It: How Family Income Predicts Children's College Chances. http://nyti .ms/1ezbuWY. (May 2015).

[2] Vincent AWMM Aleven and Kenneth R Koedinger. 2002. An effective metacognitive strategy: Learning by doing and explaining with a computer-based cognitive tutor. Cognitive Science 26, 2 (2002), 147-179.

[3] Emily Badger, Claire Cain Miller, Adam Pearce, and Kevin Quealy. 2018. Extensive Data Shows Punishing Reach of Racism for Black Boys. https://nyti.ms/2GGpFZw. (2018).

[4] Scott Bateman, Regan L Mandryk, Carl Gutwin, Aaron Genest, David McDine, and Christopher Brooks. 2010. Useful junk?: the effects of visual embellishment on comprehension and memorability of charts. In Proceedings of the SIGCHI Conference on Human Factors in Computing Systems. ACM, 2573-2582.

[5] Katerine Bielaczyc, Peter L Pirolli, and Ann L Brown. 1995. Training in self-explanation and self-regulation strategies: Investigating the effects of knowledge acquisition activities on problem solving. Cognition and instruction 13, 2 (1995), 221-252.

[6] Samuel R Bondurant, Jason M Lindo, and Isaac D Swensen. 2018. Substance abuse treatment centers and local crime. Journal of Urban Economics 104 (2018), 124-133.

[7] Michael Bostock, Vadim Ogievetsky, and Jeffrey Heer. 2011. $\mathrm{D}^{3}$ data-driven documents. IEEE Transactions on Visualization and Computer Graphics 17, 12 (2011), 2301-2309.

[8] Jeremy Boy, Francoise Detienne, and Jean-Daniel Fekete. 2015. Storytelling in information visualizations: Does it engage users to explore data?. In Proceedings of the 33rd Annual ACM Conference on Human Factors in Computing Systems. ACM, 1449-1458.

[9] Jeremy Boy, Anshul Vikram Pandey, John Emerson, Margaret Satterthwaite, Oded Nov, and Enrico Bertini. 2017. Showing people behind data: Does anthropomorphizing visualizations elicit more empathy for human rights data?. In Proceedings of the 2017 CHI Conference on Human Factors in Computing Systems. ACM, 5462-5474.

[10] Virginia Braun and Victoria Clarke. 2006. Using thematic analysis in psychology. Qualitative research in psychology 3, 2 (2006), 77-101.

[11] Giles Bruce. 2017. Study: Indiana 14th worst for drugs in U.S.

https://www . nwitimes . com/news/state-and-regional/ study-indiana-th-worst-for-drugs-in-u-s/article_ 601bd725-4992-5fe6-9a7f-81d82ac4dea9.html. (2017).

[12] Daniel Z Buchman, Pamela Leece, and Aaron Orkin. 2017. The epidemic as stigma: The bioethics of opioids. The Journal of Law, Medicine \& Ethics 45, 4 (2017), 607-620.
[13] Anne Bukten, Svetlana Skurtveit, Michael Gossop, Helge Waal, Per Stangeland, Ingrid Havnes, and Thomas Clausen. 2012. Engagement with opioid maintenance treatment and reductions in crime: a longitudinal national cohort study. Addiction 107, 2 (2012), 393-399.

[14] Alberto Cairo. 2012. The Functional Art: An introduction to information graphics and visualization. New Riders.

[15] Stuart Card, Jock Mackinlay, and Ben Shneiderman. 1999. Readings in information visualization: using vision to think. Morgan Kaufmann.

[16] In Kwon Choi, Taylor Childers, Nirmal Kumar Raveendranath, Swati Mishra, Kyle Harris, and Khairi Reda. 2019a. Concept-Driven Visual Analytics: an Exploratory Study of Model-and Hypothesis-Based Reasoning with Visualizations. In Proceedings of the 2019 CHI Conference on Human Factors in Computing Systems. ACM, 68.

[17] In Kwon Choi, Nirmal Kumar Raveendranath, Jared Westerfield, and Khairi Reda. 2019b. Visual (dis)Confirmation: Validating Models and Hypotheses with Visualizations. In 23rd International Conference in Information Visualization-Part II. IEEE, 116-121.

[18] Theodore J Cicero and Matthew S Ellis. 2015. Abuse-deterrent formulations and the prescription opioid abuse epidemic in the United States: lessons learned from OxyContin. JAMA psychiatry 72, 5 (2015), 424-430.

[19] Cristina Conati and Heather Maclaren. 2008. Exploring the role of individual differences in information visualization. In Proceedings of the working conference on Advanced visual interfaces. ACM, 199-206.

[20] Nabarun Dasgupta, Kenneth D Mandl, and John S Brownstein. 2009. Breaking the news or fueling the epidemic? Temporal association between news media report volume and opioid-related mortality. PloS one 4, 11 (2009), e7758.

[21] Peter J Davidson and Mary Howe. 2014. Beyond NIMBYism: Understanding community antipathy toward needle distribution services. International Journal of Drug Policy 25, 3 (2014), 624-632.

[22] Helen Dempster and Karen Hargrave. 2017. Understanding public attitudes towards refugees and migrants. London: Overseas Development Institute \& Chatham House (2017).

[23] Pierre Dragicevic and Yvonne Jansen. 2017. Blinded with Science or Informed by Charts? A Replication Study. IEEE Transactions on Visualization and Computer Graphics 24, 1 (2017), 781-790.

[24] Riley E Dunlap and Aaron M McCright. 2010. Climate change denial: sources, actors and strategies. In Routledge handbook of climate change and society. Routledge, 270-290. 
[25] Ana Figueiras. 2014. How to tell stories using visualization. In 2014 18th International Conference on Information Visualisation. IEEE, 18-18.

[26] C Debra M Furr-Holden, Adam J Milam, Elizabeth D Nesoff, Renee M Johnson, David O Fakunle, Jacky M Jennings, and Roland J Thorpe Jr. 2016. Not in my back yard: a comparative analysis of crime around publicly funded drug treatment centers, liquor stores, convenience stores, and corner stores in one mid-Atlantic city. Journal of studies on alcohol and drugs 77, 1 (2016), 17-24.

[27] Nahum Gershon and Ward Page. 2001. What storytelling can do for information visualization. Association for Computing Machinery. Communications of the ACM 44, 8 (2001), 31-31.

[28] Indiana State Government. 2017. INSPECT Program. https://www.in.gov/pla/inspect/2382.htm. (2017).

[29] Jonathan Gray, Lucy Chambers, and Liliana Bounegru. 2012. The data journalism handbook: how journalists can use data to improve the news. " O'Reilly Media, Inc.".

[30] Martie G Haselton, Daniel Nettle, and Damian R Murray. 2015. The evolution of cognitive bias. The handbook of evolutionary psychology (2015), 1-20.

[31] Nigel Holmes. 1984. Designer's guide to creating charts \& diagrams. Watson-Guptill.

[32] Jessica Hullman and Nick Diakopoulos. 2011. Visualization rhetoric: Framing effects in narrative visualization. IEEE Transactions on Visualization and Computer Graphics 17, 12 (2011), 2231-2240.

[33] Madeleine Keehner, Lisa Mayberry, and Martin H Fischer. 2011. Different clues from different views: The role of image format in public perceptions of neuroimaging results. Psychonomic Bulletin \& Review 18, 2 (2011), 422-428.

[34] Yea-Seul Kim, Katharina Reinecke, and Jessica Hullman. 2017. Explaining the gap: Visualizing one's predictions improves recall and comprehension of data. In Proceedings of the 2017 CHI Conference on Human Factors in Computing Systems. ACM, 1375-1386.

[35] David Klahr and Kevin Dunbar. 1988. Dual space search during scientific reasoning. Cognitive Science 12, 1 (1988), 1-48.

[36] Gary Klein, Brian Moon, and Robert R Hoffman. 2006. Making sense of sensemaking 2: A macrocognitive model. IEEE Intelligent systems 21, 5 (2006), 88-92.

[37] Gary Klein, Jennifer K Phillips, Erica L Rall, and Deborah A Peluso. 2007. A data-frame theory of sensemaking. In Expertise out of context: Proceedings of the sixth international conference on naturalistic decision making. New York, NY, USA: Lawrence Erlbaum, 113-155.

[38] Cole Nussbaumer Knaflic. 2015. Storytelling with data: A data visualization guide for business professionals. John Wiley \& Sons.
[39] Ha-Kyung Kong, Zhicheng Liu, and Karrie Karahalios. 2018. Frames and slants in titles of visualizations on controversial topics. In Proceedings of the 2018 CHI Conference on Human Factors in Computing Systems. ACM, 438.

[40] Jason Lankow, Josh Ritchie, and Ross Crooks. 2012. Infographics: The power of visual storytelling. John Wiley \& Sons.

[41] Jill H Larkin and Herbert A Simon. 1987. Why a diagram is (sometimes) worth ten thousand words. Cognitive Science 11, 1 (1987), 65-100.

[42] Bongshin Lee, Rubaiat Habib Kazi, and Greg Smith. 2013. SketchStory: Telling more engaging stories with data through freeform sketching. IEEE Transactions on Visualization and Computer Graphics 19, 12 (2013), 2416-2425.

[43] Nan Li, Dominique Brossard, Dietram A Scheufele, Paul H Wilson, and Kathleen M Rose. 2018.

Communicating data: interactive infographics, scientific data and credibility. Journal of Science Communication 17, 2 (2018), A06.

[44] Dawn L Lindsay, Holly Hagle, Piper Lincoln, Jessica Williams, and Peter F Luongo. 2017. Exploring medical students' conceptions of substance use: A follow-up evaluation. Substance abuse 38, 4 (2017), 464-467.

[45] Evan M Lowder, Bradley R Ray, and Jeffrey A Gruenewald. 2019. Criminal Justice Professionals' Attitudes Toward Mental Illness and Substance Use. Community Mental Health Journal 55, 3 (2019), 428-439.

[46] Ricardo Matheus, Marijn Janssen, and Devender Maheshwari. 2018. Data science empowering the public: Data-driven dashboards for transparent and accountable decision-making in smart cities. Government Information Quarterly (2018).

[47] Emma E McGinty, Colleen L Barry, Elizabeth M Stone, Jeff Niederdeppe, Alene Kennedy-Hendricks, Sarah Linden, and Susan G Sherman. 2018. Public support for safe consumption sites and syringe services programs to combat the opioid epidemic. Preventive Medicine 111 (2018), 73-77.

[48] Sean McKenna, N Henry Riche, Bongshin Lee, Jeremy Boy, and Miriah Meyer. 2017. Visual narrative flow: Exploring factors shaping data visualization story reading experiences. In Computer Graphics Forum, Vol. 36. Wiley Online Library, 377-387.

[49] T. Munzner. 2014. Visualization Analysis and Design. CRC Press.

[50] Yngvild Olsen and Joshua M Sharfstein. 2014. Confronting the stigma of opioid use disorder and its treatment. Jama 311, 14 (2014), 1393-1394. 
[51] Alvitta Ottley, Huahai Yang, and Remco Chang. 2015. Personality as a predictor of user strategy: How locus of control affects search strategies on tree visualizations. In Proceedings of the 33rd Annual ACM Conference on Human Factors in Computing Systems. ACM, 3251-3254.

[52] Anshul Vikram Pandey, Anjali Manivannan, Oded Nov, Margaret Satterthwaite, and Enrico Bertini. 2014. The persuasive power of data visualization. IEEE Transactions on Visualization and Computer Graphics 20, 12 (2014), 2211-2220.

[53] Larry Buchanan Haeyoun Park and Adam Pearce. 2017. You Draw It: What Got Better or Worse During Obama's Presidency. https://nyti.ms/2jS9b4b. (2017).

[54] Evan M Peck, Sofia E Ayuso, and Omar El-Etr. 2019. Data is Personal: Attitudes and Perceptions of Data Visualization in Rural Pennsylvania. In Proceedings of the 2019 CHI Conference on Human Factors in Computing Systems. ACM, 244.

[55] Ana Beatriz Nogueira Perez, Sônia Regina Zerbetto, and Angelica Martins de Souza Gonçalves. 2018. Attitudes of teachers of elementary and middle school towards the use psychoactive substances. SMAD, Revista Electrónica en Salud Mental, Alcohol y Drogas 14, 1 (2018), 45-51.

[56] Douglas L Polcin, Diane Henderson, Karen Trocki, Kristy Evans, and Fried Wittman. 2012. Community context of sober living houses. Addiction Research \& Theory 20, 6 (2012), 480-491.

[57] Nadja Popvich, Blacki Migliozzi, Josh Williams Rumsey Taylor, and Derek Watkins. 2018. How Much Hotter Is Your Hometown Than When You Were Born. https://nyti.ms/2MIIbH5. (2018).

[58] Khairi Reda, Andrew E Johnson, Michael E Papka, and Jason Leigh. 2016. Modeling and evaluating user behavior in exploratory visual analysis. Information Visualization 15, 4 (2016), 325-339.

[59] Alexander Renkl. 1997. Learning from worked-out examples: A study on individual differences. Cognitive Science 21, 1 (1997), 1-29.

[60] Tim Rhodes, Anya Sarang, Peter Vickerman, and Matthew Hickman. 2010. Policy resistance to harm reduction for drug users and potential effect of change. BMJ 341 (2010), c3439.

[61] Alison Ritter, Alison Ritter, Jacqui Cameron, Alison Ritter, and Jacqui Cameron. 2006. A review of the efficacy and effectiveness of harm reduction strategies for alcohol, tobacco and illicit drugs. Drug and Alcohol Review 25, 6 (2006), 611-624.

[62] Robin Room. 2005. Stigma, social inequality and alcohol and drug use. Drug and Alcohol Review 24, 2 (2005), 143-155.

[63] Rose A Rudd, Noah Aleshire, Jon E Zibbell, and R Matthew Gladden. 2016. Increases in drug and opioid overdose deaths-United States, 2000-2014. American Journal of Transplantation 16, 4 (2016), 1323-1327.
[64] Arvind Satyanarayan and Jeffrey Heer. 2014. Authoring narrative visualizations with ellipsis. In Computer Graphics Forum, Vol. 33. Wiley Online Library, 361-370.

[65] Edward Segel and Jeffrey Heer. 2010. Narrative visualization: Telling stories with data. IEEE Transactions on Visualization and Computer Graphics 16, 6 (2010), 1139-1148.

[66] Janet Swim, Susan Clayton, Thomas Doherty, Robert Gifford, George Howard, Joseph Reser, Paul Stern, and Elke Weber. 2009. Psychology and global climate change: Addressing a multi-faceted phenomenon and set of challenges. A report by the American Psychological Association's task force on the interface between psychology and global climate change. American Psychological Association, Washington (2009).

[67] Aner Tal and Brian Wansink. 2016. Blinded with science: Trivial graphs and formulas increase ad persuasiveness and belief in product efficacy. Public Understanding of Science 25, 1 (2016), 117-125.

[68] James J Thomas and Kristin A Cook. 2006. A visual analytics agenda. IEEE Computer Graphics \& Applications 1 (2006), 10-13.

[69] Steven Thrasher. 2018. Mike Pence Is Still to Blame for an HIV Outbreak in Indiana-but for New Reasons. https://tinyurl. com/ycrm7jkg. (Oct 2018).

[70] Chao Tong, Richard Roberts, Rita Borgo, Sean Walton, Robert Laramee, Kodzo Wegba, Aidong Lu, Yun Wang, Huamin Qu, Qiong Luo, and others. 2018. Storytelling and visualization: An extended survey. Information 9, 3 (2018), 65.

[71] Susan Trickett and Gregory Trafton. 2007. A primer on verbal protocol analysis. In Handbook of virtual environment training, J. Cohn D. Schmorrow and Nicholson (Eds.). Chapter 18.

[72] Heather Barnes Truelove and Craig Parks. 2012. Perceptions of behaviors that cause and mitigate global warming and intentions to perform these behaviors. Journal of Environmental Psychology 32, 3 (2012), 246-259.

[73] Edward R Tufte. 2001. The Visual Display of Quantitative Information (2 ed.). Graphics Press.

[74] Zezhong Wang, Shunming Wang, Matteo Farinella, Dave Murray-Rust, Nathalie Henry Riche, and Benjamin Bach. 2019. Comparing Effectiveness and Engagement of Data Comics and Infographics. In Proceedings of the 2019 CHI Conference on Human Factors in Computing Systems. ACM, 253.

[75] Hazel Watson, William Maclaren, and Susan Kerr. 2007. Staff attitudes towards working with drug users: development of the Drug Problems Perceptions Questionnaire. Addiction 102, 2 (2007), 206-215. 
[76] H Watson, W Maclaren, F Shaw, and A Nolan. 2003. Measuring staff attitudes to people with drug problems: The development of a tool. Glasgow, Scotland: Scottish Executive Drug Misuse Research Programme (2003).

[77] Zhenpeng Zhao, Rachael Marr, and Niklas Elmqvist. 2015. Data comics: Sequential art for data-driven storytelling. Technical Report, University of Maryland (2015).
[78] Qiyu Zhi, Alvitta Ottley, and Ronald Metoyer. 2019. Linking and Layout: Exploring the Integration of Text and Visualization in Storytelling. In Computer Graphics Forum, Vol. 38. Wiley Online Library, 675-685.

[79] Caroline Ziemkiewicz, Alvitta Ottley, R Jordan Crouser, Krysta Chauncey, Sara L Su, and Remco Chang. 2012. Understanding visualization by understanding individual users. IEEE Computer Graphics \& Applications 32, 6 (2012), 88-94. 